\title{
Proposta de modelagem não-linear do desempenho germinativo de sementes de milho híbrido
}

\author{
Nonlinear proposal modeling of seed germination performance of hybrid corn seeds
}

\author{
Sebastião Gazola ${ }^{*}$ Carlos Alberto Scapim ${ }^{I I}$ Terezinha Aparecida Guedes ${ }^{\mathrm{I}}$ \\ Alessandro de Lucca e Braccini"
}

\section{RESUMO}

Este trabalho foi desenvolvido com o objetivo de ajustar um modelo de regressão não-linear para estimar o desempenho germinativo de sementes de três lotes comerciais de milho híbrido OC 705, submetidas ao teste de envelhecimento acelerado, à temperatura de $43^{\circ} \mathrm{C}$. Utilizou-se o modelo logístico $y(t)=C /(1+\exp (B(t-M)))$ para ajustar os dados. As medidas de viés de Box e de curvaturas paramétrica e intrínseca foram usadas para o diagnóstico do modelo. O modelo proposto ajusta-se adequadamente aos dados de percentuais germinativos de sementes de milho híbrido OC 705, para todos os lotes. Com base nas estimativas dos parâmetros e qualidade de ajuste, o lote três foi identificado como sendo o melhor para comercialização, por apresentar menor redução no poder germinativo ao longo do tempo.

Palavras-chave: Zea mays L., viabilidade de semente, ajuste de percentual germinativo, regressão nãolinear, envelhecimento acelerado.

\section{ABSTRACT}

This research was developed with the goal to adjust a nonlinear regression model to estimate seed germination performance of three commercial seed lots of hybrid maize OC 705 , subjected to accelerated aging test at a temperature of $43^{\circ} \mathrm{C}$. The logistic model $y(t)=C /(1+\exp (B(t-M)))$ was used to fit the data. Measures of bias Box and parametric intrinsic bends were used for the diagnosis of the model. The proposed model fits properly to seed germination percentage data of hybrid corn seeds for OC 705, for all seed lots. Based on estimates of the parameters and fit quality, the seed lot three was identified as being the best for marketing, by presenting less reduction in seed germination power over time.

Key words: Zea mays L., seed viability, germination percentage adjustment, nonlinear regression, accelerated aging.

\section{INTRODUÇÃO}

É muito importante prever os efeitos do armazenamento das sementes para que se tenha uma perda suave da viabilidade (ELLIS \& ROBERTS, 1980). A perda de viabilidade e vigor é mais rápida principalmente sob condições tropicais (ANDREOLI $\&$ ANDRADE, 2007). Fatores como a temperatura, umidade relativa do ar e a qualidade fisiológica inicial das sementes contribuem para essas perdas durante $o$ armazenamento (ROBERTS, 1960). Estimativas de germinação seguras são importantes para a manutenção de germoplasma e para os mantenedores de sementes comerciais, tanto na produção quanto no armazenamento (MEAD \& GRAY, 1999). Equações capazes de estimar a viabilidade foram desenvolvidas inicialmente por ROBERTS (1960, 1961a, 1961b e 1973) e, posteriormente, por ELLIS \& ROBERTS (1980), que são atualmente usadas por muitos pesquisadores.

ANDREOLI (2004) descreveu uma simplificação da equação básica $\log p_{50}=\mathrm{K}_{\mathrm{v}}-\mathrm{C}_{1} \mathrm{~m}-\mathrm{C}_{2} \mathrm{t}$ de ROBERTS (1960) e da equação $\mathrm{v}=\mathrm{K}_{\mathrm{i}}-\mathrm{p} / 10^{\mathrm{K}_{c}-\mathrm{C}_{\mathrm{w}} \operatorname{logm}-\mathrm{C}_{\mathrm{H}} \mathrm{H}_{\mathrm{C}} \mathrm{C}_{\mathrm{t}} \mathrm{t}^{2}}$ de ELLIS \& ROBERTS (1980) para melhorar a precisão nas estimativas de viabilidade, dada por $\mathrm{Vp}=\operatorname{Vi-\operatorname {tg}(\beta )}$.p, em que Vp é a viabilidade em probito no período p, Vi é a viabilidade inicial e tg(ß) é a taxa de deterioração.

A transformação dos percentuais germinativos em probitos gera um novo conjunto de dados que tem comportamento aproximadamente linear, possibilitando que sejam ajustados pela equação linear

'Departamento de Estatística, Universidade Estadual de Maringá (UEM), Av. Colombo, 5790, 87020-900, Maringá, PR, Brasil. E-mail: sgazola@uem.br.*Autor para correspondência.

IIDepartamento de Agronomia, UEM, Maringá, PR, Brasil. 
$\mathrm{Vp}=\mathrm{Vi}-\operatorname{tg}(\beta)$.p, que é simples e de fácil interpretação (ANDREOLI, 2004). No entanto, nem sempre se consegue a linearização dos dados e se torna necessário buscar outros modelos. Nestas situações, não é possível descrever um fenômeno por meio dos modelos lineares e os parâmetros não são possíveis de serem estimados com fórmulas analíticas. Processos como crescimento, nascimento, mortalidade e rendimento raramente se relacionam linearmente com as variáveis exploratórias.

Em casos desse tipo, os modelos nãolineares apresentam-se como uma boa opção e são aplicados em biologia, engenharia florestal, zoologia e ecologia, com os quais é possível modelar o crescimento ocorrido em vegetais, animais e também em seres humanos (DRAPER \& SMITH, 1981).

GUIMARÃES et al. (2006) ajustou modelos de regressão não-lineares para estudar a curva de lactação de caprinos leiteiros, produção de leite ao longo do tempo de lactação e, dentre os modelos estudados, o modelo $\mathrm{Y}=\mathrm{t} / \mathrm{a}+\mathrm{bt}+\mathrm{ct}^{2}$ foi o que apresentou o melhor ajuste.

Para ajustar valores de percentuais de germinação em função do tempo (em dias) para métodos de superação de dormência, GASPAR-OLIVEIRA et al. (2008) ajustaram o modelo não-linear de Gompertz $\mathrm{Y}=\exp (\alpha-\exp (\beta+\gamma \cdot x))$ que proporcionou ajuste adequado aos dados ( $\mathrm{R}^{2}$ próximo de 1$)$.

CALHA et al. (2008) estudaram a influência da idade na germinação das sementes de Alisma plantago-aquatica e o regime de temperatura mais adequado à germinação por meio da regressão não-linear, ajustando o modelo log$\log$ ístico, $\mathrm{Y}=\mathrm{M} /(1+\exp ((\mathrm{T} 50-\mathrm{x}) / \mathrm{c}$.

MAIA et al. (2009) utilizaram modelos nãolineares para estudar as curvas de crescimento ao longo do tempo de duas variedades de bananeiras submetidas a cinco doses de um regulador de crescimento. Dentre os modelos ajustados, o modelo logístico proporcionou o melhor ajuste e este foi utilizado para descrever as curvas.

Em estudos envolvendo curvas de crescimento de dois cultivares de feijoeiro, MARTINS FILHO et al. (2008) utilizaram a metodologia Bayesiana e ajustaram o modelo logístico. Obtiveram resultados eficientes ao testar a hipótese de igualdade dos parâmetros mediante a avaliação do intervalo de credibilidade que permitiu a comparação dos dois cultivares.

MENDES et al. (2008), no estudo do crescimento de fêmeas da raça Hereford, concluíram que o modelo logístico difásico proporcionou o melhor ajuste, recomendando esse modelo para descrever a curva de crescimento em questão.
MAZZINI et al. (2005) ajustaram os modelos não-lineares a dados de crescimento de novilhos Hereford e concluíram que os modelos de Gompertz e von Bertalanffy tiveram melhor qualidade de ajuste, indicando esses modelos para descrever o crescimento de novilhos até dois anos.

Os modelos não-lineares apresentam algumas vantagens em relação aos lineares, tais como a origem na teoria e princípios biológicos, físicos e químicos e aplicados em situações que podem ser descritas por um menor número de parâmetros. Entretanto, existem desvantagens, já que requerem algoritmos iterativos de ajuste, uso de estimativas iniciais para o ajuste dos parâmetros, permitem apenas aproximações em vez de inferências mais precisas e requerem sólida compreensão acerca do fenômeno em estudo (SCHABENBERGER \& PIERCE, 2002).

Este estudo visa a conhecer a distribuição do percentual de germinação obtido sob condição de estresse para diferentes lotes de sementes de milho híbrido e com diferentes tempos de armazenamento. Nesse sentido, as empresas produtoras de sementes poderiam avaliar a qualidade das sementes ao longo do tempo de armazenamento. O objetivo deste trabalho foi ajustar um modelo de regressão não-linear para estimar, em três épocas distintas, a viabilidade de sementes de milho híbrido, em função do tempo de envelhecimento acelerado.

\section{MATERIAL E MÉTODOS}

O experimento foi realizado no Laboratório de Sementes, na Fazenda Experimental de Iguatemi, pertencente ao CCA/UEM, em Maringá, PR. Foram utilizados três lotes de sementes de milho híbrido OC 705. Para todos os lotes, a porcentagem de germinação foi avaliada em janeiro e julho de 2003 e abril de 2004. Foram obtidos nove conjuntos de dados provenientes de três lotes em três avaliações e denotados por L11, L12,L13, L21, L22, L23, L31,L32 eL33, em que o primeiro número refere-se ao lote e o segundo à avaliação. Os dados foram obtidos de percentuais germinativos de sementes de milho submetidas ao teste de envelhecimento acelerado em temperatura de $43^{\circ} \mathrm{C}$. Os testes de germinação foram realizados à temperatura de $30^{\circ} \mathrm{C}$ com cinco repetições de 200 sementes. Observou-se o percentual de germinação inicial (tempo zero) e, nas condições de estresse, durante $24,48,72$, $96,120,144,168$ e 192 horas de envelhecimento acelerado.

Para o estudo das curvas dos percentuais germinativos ao longo tempo de estresse, utilizou-se o software SAS 9.1.3 (2002) e ajustou-se o modelo de 
regressão não-linear logístico, que apresenta uma curva assintótica, descrito por:

$\mathrm{Y}=\mathrm{V}_{\mathrm{i}} /(1+\exp (\mathrm{B}(\mathrm{t}-\mathrm{M})))+\varepsilon,(1)$ em que: Vi representa a viabilidade inicial da semente, determinada por meio de testes de germinação, o parâmetro B representa a taxa de decrescimento ao longo do tempo, o parâmetro $\mathrm{M}$ indica o tempo no qual o percentual de germinação atinge aproximadamente $50 \%$ e $\varepsilon$ representa o erro aleatório (RATKOWSKY, 1983).

Um modelo não-linear é considerado o melhor, em comparação a outros, se ele apresentar o comportamento mais linear. Para avaliar a nãolinearidade de um modelo, as ferramentas utilizadas são as medidas de curvatura de Bates e Watts e o viés de box.

BATES \& WATTS (1988) descrevem formalmente as expressões para o cálculo das curvaturas paramétricas e intrínsecas, que servem para estudar a presença de não-linearidade em um modelo. A ideia é que, quanto mais linearizável for a função resposta, melhor serão os resultados inferenciais associados ao modelo não-linear. Em princípio, o modelo é melhor quanto menor forem as curvaturas. Segundo SOUZA (1998), o valor limite de curvatura sugerido por Bates e Watts é dado por $1 / 2 \sqrt{\mathrm{F}_{1-\alpha,(p, n-p)}}$. $\mathrm{O}$ alto valor da curvatura intrínseca indica a intensidade da não-linearidade da função resposta e o alto valor da curvatura paramétrica indica qual ou quais parâmetros do modelo são responsáveis pelo afastamento da forma linear. A outra medida importante é o viés de box, que é calculado para cada parâmetro e permite a identificação dos que são responsáveis pela não-linearidade, ou seja, o viés de box ajuda a detectar quais os parâmetros que individualmente são responsáveis pelo excesso de curvatura. RATKOWSKY (1983) sugere como valor padrão para a determinação da não-linearidade o valor de $1 \%(0,01)$, obtido pela razão entre o valor absoluto do quociente do viés e o valor do parâmetro.

Ainda buscando avaliar a qualidade do ajuste, bandas de confiança podem ser construídas utilizando-se a estatística t de Student (BATES \& WATTS, 1988).

\section{RESULTADOS E DISCUSSÃO}

O modelo proposto (equação 1), fixado o valor da germinação inicial Vi, específico para cada conjunto de dados, ajustou-se adequadamente a todos os conjuntos de dados, conforme mostram os valores de curvaturas e do viés de box (Tabela 1). Os valores de germinação estimados e observados, são apresentados nas figuras 1,2 e 3 .

As estimativas do parâmetro B, que representa a taxa de deterioração, indicam a perda dos percentuais germinativos ao longo do tempo de envelhecimento acelerado. $\mathrm{O}$ lote três, ao longo do tempo de armazenamento, épocas de janeiro e julho de 2003 e abril de 2004, manteve, aproximadamente, a mesma taxa de deterioração. O lote dois teve taxa de deterioração aproximadamente duplicada da primeira para a terceira época. O lote um teve o maior aumento da taxa de deterioração ao longo do tempo de armazenamento, de 0,025 na época um para 0,111 na época três. Esse lote aumentou em mais de quatro vezes

Tabela 1 - Estimativas dos parâmetros, viés e curvaturas paramétrica e intrínseca da equação não-linear $\mathrm{y}=\mathrm{V}_{\mathrm{i}} /(1+\exp (\mathrm{B}(\mathrm{t}-\mathrm{M})))$, ajustada aos dados de germinação de sementes de milho OC 705 para os três lotes, em três épocas de avaliação.

\begin{tabular}{|c|c|c|c|c|c|c|c|c|c|c|}
\hline & & \multicolumn{3}{|c|}{--------------'Lote 1-------------- } & \multicolumn{3}{|c|}{ 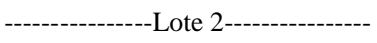 } & \multicolumn{3}{|c|}{ - } \\
\hline & & $\mathrm{Jan} / 03$ & $\mathrm{Jul} / 03$ & $\mathrm{Abr} / 04$ & $\mathrm{Jan} / 03$ & $\mathrm{Jul} / 03$ & $\mathrm{Abr} / 04$ & $\mathrm{Jan} / 03$ & $\mathrm{Jul} / 03$ & $\mathrm{Abr} / 04$ \\
\hline \multirow{3}{*}{$\begin{array}{l}\text { Parâmetros } \\
\text { Estimados }\end{array}$} & $\mathrm{V}_{\mathrm{i}}^{*}$ & 0,955 & 0,953 & 0,886 & 0,966 & 0,947 & 0,939 & 0,933 & 0,928 & 0,911 \\
\hline & B & 0,025 & 0,044 & 0,111 & 0,025 & 0,039 & 0,046 & 0,056 & 0,044 & 0,052 \\
\hline & M & 147 & 115 & 88 & 171 & 142 & 72 & 124 & 110 & 104 \\
\hline \multirow{2}{*}{ Viés } & $\mathrm{B}$ & 0,004 & 0,002 & 0,014 & 0,002 & 0,006 & 0,014 & 0,002 & 0,003 & 0,003 \\
\hline & M & 0,000 & 0,000 & 0,000 & 0,000 & 0,000 & 0,000 & 0,000 & 0,000 & 0,000 \\
\hline \multicolumn{2}{|c|}{ Curv. Paramétrica } & 0,143 & 0,101 & 0,281 & 0,168 & 0,156 & 0,253 & 0,095 & 0,123 & 0,128 \\
\hline \multicolumn{2}{|c|}{ Curv. Intrínseca } & 0,095 & 0,067 & 0,150 & 0,086 & 0,115 & 0,168 & 0,063 & 0,082 & 0,088 \\
\hline \multicolumn{2}{|l|}{ SQE } & 0,264 & 0,096 & 0,209 & 0,135 & 0,283 & 0,555 & 0,061 & 0,132 & 0,112 \\
\hline
\end{tabular}

$\mathrm{V}_{\mathrm{i}}{ }^{*}=$ valor da viabilidade inicial fixado para cada conjunto de dados, que corresponde ao parâmetro $\mathrm{C}$.

Valor limite para o viés de box $=0,01$.

Valor limite para a curvatura $=0,26$.

Curv. Curvatura.

SQE soma do quadrado do erro.

Nota: Os valores do viés para o parâmetro M são inferiores a 0,0001.

Ciência Rural, v.41, n.4, abr, 2011. 

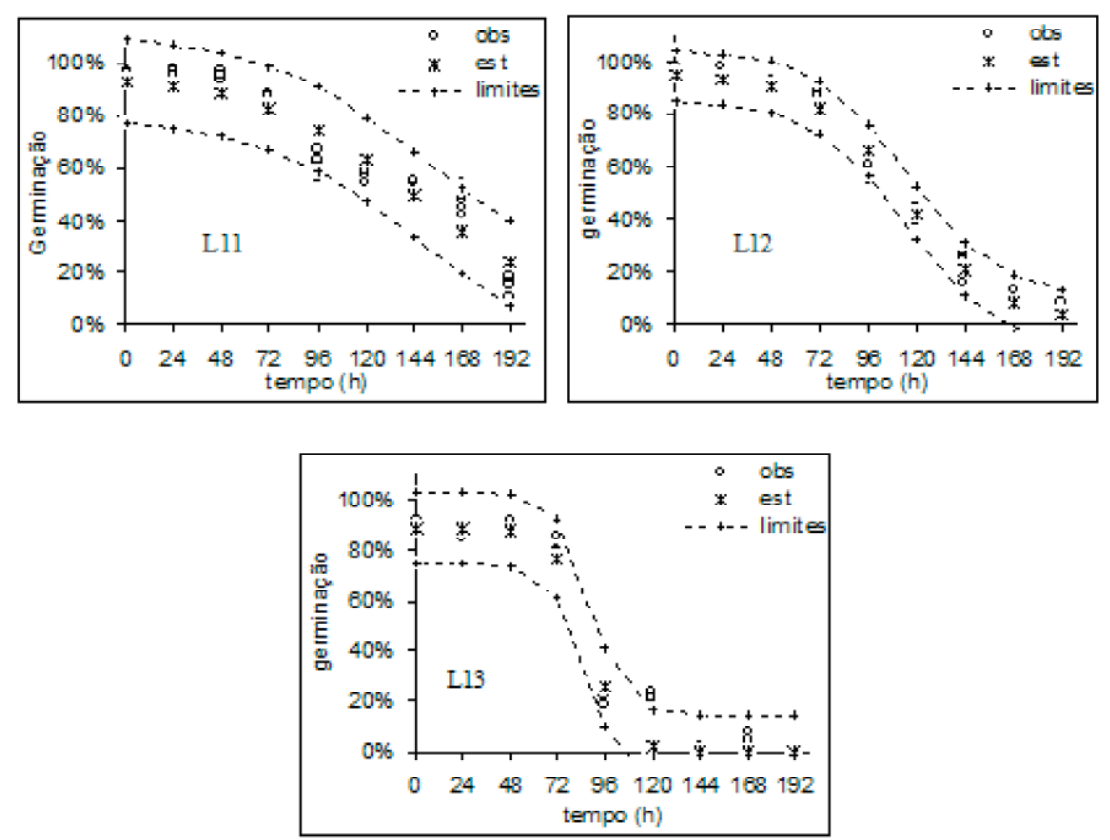

Figura 1 - Valores de germinação observados (o) e estimados pela equação 2 (*) e intervalo de confiança (--), para as três avaliações do lote 1 (L11, L12 e L13), em função do tempo de envelhecimento de sementes de milho híbrido OC 705.
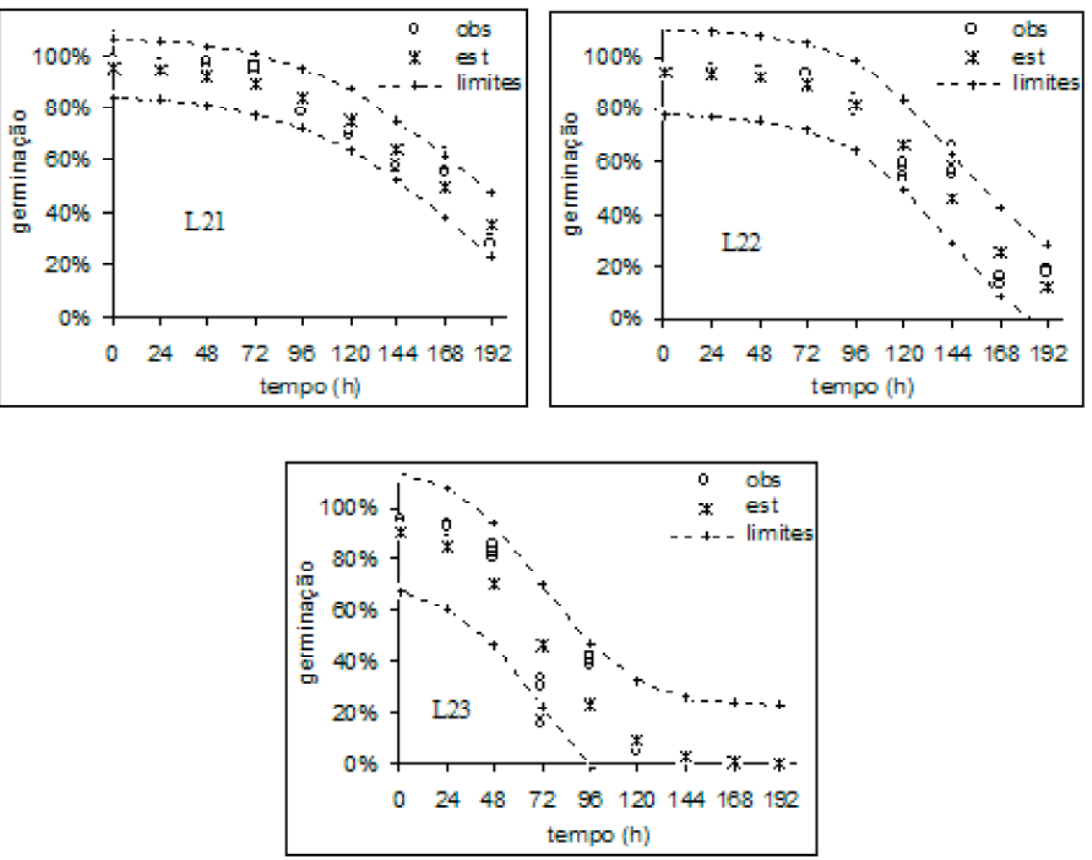

Figura 2 - Valores de germinação observados (o) e estimados pela equação 2 (*) e intervalo de confiança (--), para as três avaliações do lote 2 (L21, L22 e L23), em função do tempo de envelhecimento de sementes de milho híbrido OC 705. 


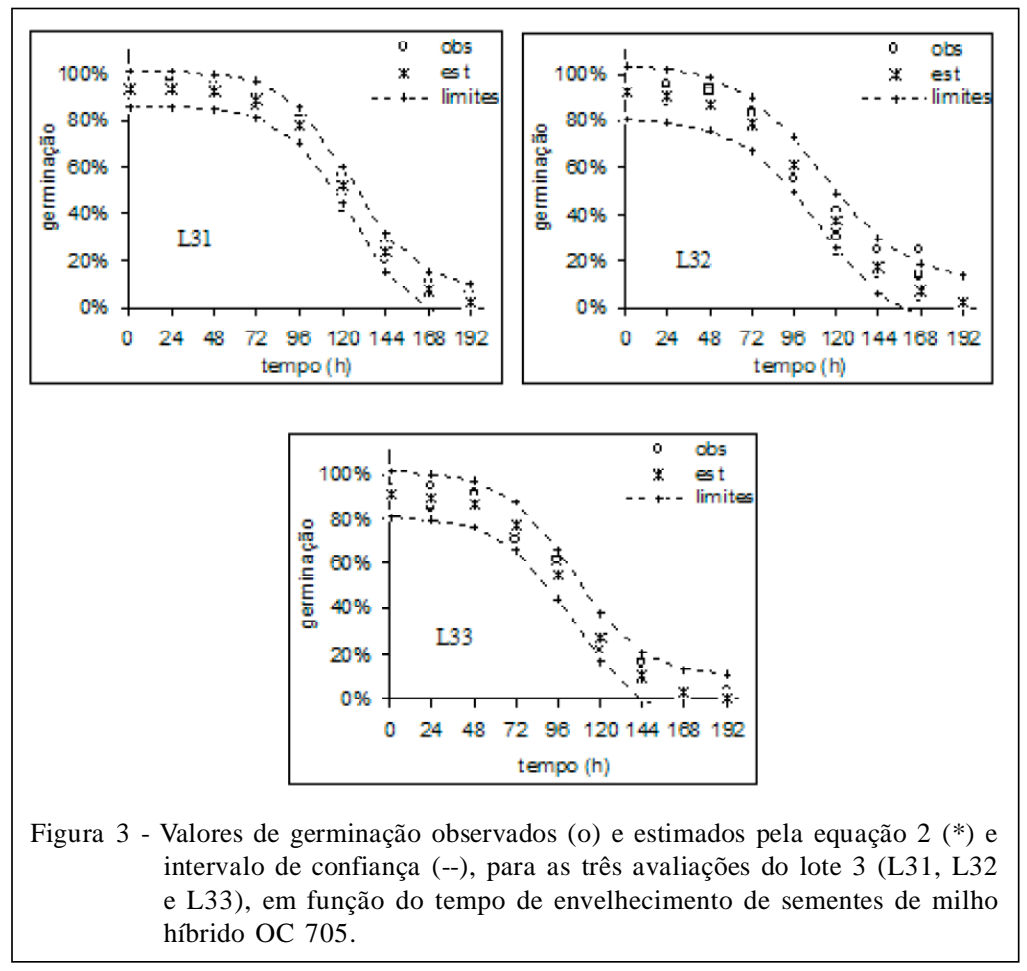

ao longo do tempo de armazenamento (Tabela 1 e Figuras 1, 2 e 3).

O parâmetro M indica o tempo aproximado em que o lote atingiu um percentual de germinação de aproximadamente $50 \%$ do máximo. Os lotes um e dois tiveram uma variação muito grande deste tempo nas três avaliações. Para o lote um, variou de 147 dias na época de janeiro de 2003 para 88 dias na época de abril de 2004. Para o lote dois, variou de 171 dias na época de janeiro de 2003 para 72 dias na época de abril de 2004. O lote três manteve este tempo semelhante, 104, 110 e 124 horas, ao longo do tempo de armazenamento, épocas de janeiro e julho de 2003 e abril de 2004 (Tabela 1 e Figuras 1, 2 e 3).

Para todos os lotes e em todas as épocas, a medida de curvatura intrínseca indicou forte presença de linearidade. O lote três, ao longo do tempo de armazenamento, teve as menores medidas de curvatura intrínseca, indicando melhor qualidade de ajuste que os lotes dois e três (BATES \& WATTS, 1988) (Tabela 1). As medidas de curvatura paramétrica foram não significativas, isto porque não houve parâmetro ou parâmetros responsáveis por fuga da linearidade. De fato, o viés de box teve valores inferiores ao valor limite de 0,01 para todos os parâmetros. Observou-se que o lote três teve as menores medidas de curvatura intrínseca e por sua vez das médias do viés de box, nas três épocas (BATES \& WATTS, 1988) (Tabela 1). Os lotes um e dois, na época abril de 2004, tiveram os maiores valores de medida de curvatura intrínseca e paramétrica, apesar de estarem abaixo do valor limite de 0,26. Ainda, seus valores do viés de box, ultrapassaram, ainda que levemente, o valor limite de 0,01 (Tabela 1) para o parâmetro B.

Para verificar a normalidade dos erros, foi aplicado o teste de Kolmogorov-Smirnov que não rejeitou esta hipótese em nível de 5\% de significância ( $\mathrm{P}$-valor $>0,05$ ) e, pelas figuras 1,2 e 3 , pode-se observar que os valores estimados estão dentro das bandas para $95 \%$ de confiança.

Os dados de percentuais germinativos que representam curvas sigmoidais decrescentes, sendo que o percentual inicial de germinação, Vi, é o máximo de germinação, decrescendo ao longo do tempo, ajustam-se adequadamente ao novo modelo. $\mathrm{O}$ novo modelo ajustou-se adequadamente a todos os lotes. A incorporação da constante Vi foi importante para a qualidade do ajuste, tornou o modelo logístico simples e de fácil interpretação, pois $\mathrm{Vi}, \mathrm{B}$ e $\mathrm{M}$ fornecem informações importantes a respeito do percentual germinativo de sementes.

Com base nas discussões dos resultados, realizadas por meio das médias de curvatura, viés de box, soma do quadrado do erro e interpretação dos parâmetros estimados B e M, é possível afirmar que o lote três é o que teve o melhor ajuste. Este lote teve os 
menores valores do viés de box, menores medidas de curvatura e menor valor da SQE. Ainda, teve tempo de queda do percentual de germinação para 50\%, semelhante nas três épocas, e taxa de deterioração aproximadamente constante nas três épocas. Pode-se, então, indicar o lote três como sendo o lote de melhor qualidade.

\section{CONCLUSÃO}

O modelo logístico proposto com a constante de viabilidade inicial, $\mathrm{V}_{\mathrm{i}}$, ajusta-se, adequadamente, a dados de percentuais germinativos de sementes de milho híbrido OC 705.

Ao longo do tempo de armazenamento, o lote 3 apresentou perda aproximadamente constante do percentual germinativo e tempo semelhante em que o percentual de germinação cai para $50 \%$, sendo, portanto, o lote mais indicado para a comercialização.

\section{REFERÊNCIAS}

ANDREOLI, C. Simplificação da equação de viabilidade para predizer a viabilidade de sementes de milho e soja. Pesquisa Agropecuária Brasileira, v.39, n.9, p.911-917, 2004. Disponível em: <http:// www.scielo.br/scielo.php? script $=$ sci_arttext\&pid $=$ S0 $100-$ 204X2004000900011>. Acesso em: 31 jan. 2011. doi: 10.1590/ S0100-204X2004000900011.

ANDREOLI, C.; ANDRADE, R.V. Seed longevity to predict viability of corn seed during open storage. Revista Brasileira de Milho e Sorgo, v.6, n.2, p.247-255, 2007. Disponível em: <http://rbms.cnpms.embrapa.br/index.php/ojs/article/view/ 229>. Acesso em: 31 jan. 2011.

BATES, D.M.; WATTS, D.G. Nonlinear regression analysis and its applications. New York: John Wiley \& Sons, 1988. $365 \mathrm{p}$.

CALHA, I. et al. Germinação de sementes de populações de orelha-de-mula (Alisma plantago-aquatica) resistentes e susceptíveis ao bensulfurão-metilo. Revista de Ciências Agrárias, v.31, n.1, p.117-130, 2008. Disponível em: <http:/ /www.scielo.oces.mctes.pt/pdf/rca/v31n1/v31n1a12>. Acesso em: 31 jan. 2011.

DRAPER, N.R.; SMITH, H. Applied regression analysis. 2.ed. New York: John Wiley \& Sons, 1981. 710p.

ELLIS, R.H.; ROBERTS, E.H. Improved equations for the prediction of seed longevity. Annals of Botany, v.45, n.1, p.13-30, 1980. Disponível em: <http://aob.oxfordjournals.org/ content/45/1/13>. Acesso em: 31 jan. 2011.

GASPAR-OLIVEIRA, C.M. et al. Duração do teste de germinação de Brachiaria brizantha cv. 'Marandu' (hochst. ex a. rich.) stapf1. Revista Brasileira de Sementes, v.30, n.3, p.30-38, 2008. Disponível em: <http://www.scielo.br/ scielo.php?script =s ci_arttext \& pid=S 0101 $31222008000300005 \& \operatorname{lng}=p t \& n r m=i s o>$. Acesso em: 31 jan. 2011. doi: 10.1590/S0101-31222008000300005.

GUIMARÃES, V.P. et al. Utilização de funções matemáticas no estudo da curva de lactação em caprinos. Revista Brasileira de Zootecnia, v.35, n.2, p.535-543, 2006. Disponível em $<$ http://www.scielo.br/scielo.php?script=sci_arttext\&pid=S1516$35982006000200028 \& \operatorname{lng}=$ pt\&nrm=iso $>$. Acesso em: 31 jan. 2011. doi: $10.1590 / \mathrm{S} 1516-35982006000200028$

MAIA, E. et al. Método de comparação de modelos de regressão não-lineares em bananeiras. Ciência Rural, v.39, n.5, p.1380-1386, 2009. Disponível em: <http://www.scielo.br/ scielo.php? script $=$ sci_art text \& pid = S $0103-$ $84782009000500012 \& \operatorname{lng}=$ pt\&nrm=iso>. Acesso em: 31 jan. 2011 .

MARTINS FILLHO, S. et al. Abordagem bayesiana das curvas de crescimento de duas cultivares de feijoeiro. Ciência Rural, v.38, n.6, p.1516-1521, 2008. Disponível em: <http:// www.scielo.br/scielo.php? script $=$ sci_arttext\&pid=S0 103 $84782008000600004 \& \operatorname{lng}=$ pt\&nrm $=$ iso $>$. Acesso em: 31 jan. 2011. doi: $10.1590 / \mathrm{S} 0103-84782008000600004$.

MAZZINI, A. R. A. et al. Curva de crescimento de novilhos Hereford: heterocedasticidade e resíduos autorregressivos. Ciência Rural, v.35, n.2, p.422-427, 2005. Disponível em: $<$ http://www.scielo.br/scielo.php?script=sci_arttext\&pid=S0103$84782005000200028 \& \operatorname{lng}=$ pt\&nrm=iso $>$. Acesso em: 31 jan. 2011. doi: $10.1590 / \mathrm{S} 0103-84782005000200028$.

MEAD, A.; GRAY, D. Prediction of seed longevity: a modification of shape of the Ellis and Roberts seed survival curves. Seed Science Research, v.9, p.63-73, 1999. Disponível em: <http://journals.cambridge.org/action/ displayFulltext?type $=1 \&$ fid $=693324 \&$ jid=SSR \&volumeId=9\&i ssueId=01\&aid=693316>. Acesso em: 31 jan. 2011.

MENDES, P. M. et al. Modelo logístico difásico no estudo do crescimento de fêmeas da raça Hereford. Ciência Rural, v.38, n. 7 p.1984-1990, 2008. Disponível em: <http:// www.scielo.br/scielo.php? script $=$ sci_arttext\&pid=S0103$84782008000700029 \& \operatorname{lng}=p t \& n r m=i s o>$. Acesso em: 31 jan. 2011. doi: $10.1590 / \mathrm{S} 0103-84782008000700029$.

RATKOWSKY, D.A. Nonlinear regression modeling. New York: Marcel Dekker, 1983. 276p.

ROBERTS, E.H. The viability of cereal seed in relation to temperature and moisture. Annals of Botany, v.24, n.93, p.12-31, 1960.

ROBERTS, E.H. Viability of cereal seed for brief and extended periods. Annals of Botany, v.25, n.99, p.373-380, 1961a.

ROBERTS, E.H. Viability of rice seed in relation to temperature, moisture content, and gaseous environment. Annals of Botany, v. 25 n, 99, p.381-390, 1961b.

ROBERTS, E.H. Predicting the storage life of seeds. Seed Science \& Technology, v.1 n.3, p.499-514, 1973.

SAS institute. SAS/STAT User's guide, versão 9.1.3. Cary, NC, 2002. 748p. Disponível em: <http://www.google.com.br/ \#hl=pt-BR\&biw=1003\&bih=615\&q=SAS+institute.++SAS\%2F STAT+User\%E2\%80\%99s+guide $\% 2 \mathrm{C}+$ vers $\% \mathrm{C} 3 \% \mathrm{~A} 3 \mathrm{o}+9.1 .3 .+\mathrm{C}$ ary $\% 2 \mathrm{C}+\mathrm{NC} \% 2 \mathrm{C}+20022003 .++748 \mathrm{p} \& \mathrm{aq}=\mathrm{f} \& \mathrm{aqi}=\& \mathrm{aql}=\& \mathrm{oq}=\& \mathrm{fp}=$ 1f83e47f66a57597>. Acesso em: 31 jan. 2011.

SCHABENBERGER, O.; PIERCE, F.J. Contemporary statistical models. Boca Raton: CRC, LLC, 2002. 738p.

SOUZA, G.S. Introdução aos modelos de regressão linear e não-linear. Brasília: Embrapa, 1998. 505p. 